\title{
Análisis econométrico \\ de la inversión privada en Brasil
}

\section{Marcio Bruno Ribeiro \\ marciob@ucb.br \\ Universidad Católica \\ de Brasilia \\ Joanilio Rodolpho \\ Teixeira \\ joanilioteixeira@hotmail.com \\ Departamento de Economía, \\ Universidad de Brasilia}

Este artículo analiza los principales factores determinantes de la inversión privada en Brasil durante el período 1956-1996, a través de un modelo empírico utilizado en los estudios más recientes sobre países en desarrollo. Los procedimientos econométricos, además de tomar en cuenta la no estacionariedad de las series de datos que se examinan, consideran las posibles dificultades vinculadas con tratar las variables condicionantes como exógenas o como instrumentos de política. Los resultados muestran, tanto en las ecuaciones de largo plazo como en los modelos de corto plazo, el impacto positivo de las variables producto, inversión pública y crédito financiero y el efecto negativo del tipo de cambio. Los resultados de los tests de exogeneidad débil y superexogeneidad ponen en evidencia la importancia de la inversión pública y el crédito como instrumentos de política económica, y permiten obviar la crítica de Lucas. 


\section{I}

\section{Introducción}

Las decisiones de inversión en bienes de capital son de extrema importancia para el crecimiento económico de un país y están relacionadas generalmente con el sector privado, del cual se espera que desempeñe un papel fundamental en la realización de inversiones productivas.

En los países en desarrollo, la reducción de las tasas de inversión a partir de los años ochenta motivó la investigación empírica de los determinantes principales de la inversión privada. Otro factor motivador fue la presencia de los elementos institucionales y estructurales que caracterizaron la formación de capital en esos países, como represión financiera en el mercado crediticio, fuerte presencia del gobierno, dependencia de las divisas e inestabilidades económicas. Los estudios más recientes sobre la inversión del sector privado en los países en desarrollo (entre otros, Greene y Villanueva,1995; Servén y Solimano, 1993; Agosin, 1994) incorporaron además al análisis empírico variables representativas de las incertidumbres en la decisión de invertir y de las restricciones externas. Estas últimas, debido a la crisis de la deuda externa y el deterioro de las relaciones de intercambio que caracterizaron a las economías en desarrollo durante los años ochenta.

En el caso específico de Brasil, los estudios empíricos trataron de analizar principalmente las relaciones entre las inversiones de los sectores privado y público. El estudio más reciente en esta línea de investigación es el trabajo de Cruz y Teixeira (1999). Utilizando los tests de estacionariedad y cointegración, procedimientos que permiten la realización de estimaciones considerando la no estacionariedad de series cronológicas, los resultados obtenidos mostraron que las inversiones pública y privada son complementarias en el largo plazo y sustitutivas en el corto plazo.

No obstante, lo que viene a motivar el presente estudio es el hecho de que el análisis empírico de la

$\square$ Los autores agradecen al Consejo nacional de desarrollo científico y tecnológico (CNPq) y a la Coordinación del perfeccionamiento del personal de la enseñanza superior (CAPES) el apoyo prestado a esta investigación, y agradecen también los comentarios de Francisco G. Carneiro, Manuel R. Agosin y Oscar Altimir. Naturalmente, los autores son los únicos responsables del contenido de este artículo. inversión privada no puede considerarse aún plenamente satisfactorio desde la perspectiva de la econometría moderna. Ni siquiera en los estudios empíricos más recientes, sea para Brasil o para un conjunto de países en desarrollo, se investigó el carácter exógeno de las variables explicativas, lo que significa que la inversión privada se consideró, a priori, una variable típicamente endógena. Dado que la estimación de los parámetros de un modelo econométrico se realiza condicionada a sus variables explicativas, se supone directamente que el proceso marginal de cada una de ellas carece de información relevante para la estimación. Sin embargo, estas hipótesis deben ser verificadas empíricamente. En caso de que el proceso marginal de alguna variable explicativa se muestre relevante, ésta no podrá considerarse exógena, pues estaríamos desestimando informaciones importantes. Por consiguiente, esto nos llevaría a una estimación ineficiente de los parámetros.

Otra utilidad de los tests empíricos de exogeneidad para el tema que nos ocupa reside en la identificación de las variables macroeconómicas que pueden utilizarse como instrumentos de política; o sea, de aquellas cuyos cambios de estructura no afectan los parámetros del modelo. Así, no estaríamos sujetos a la crítica de Lucas (1976), pues ésta sostiene que, bajo la hipótesis de expectativas racionales, los parámetros estimados a partir de un modelo econométrico no se mantendrían. La ocurrencia de cambios de política llevaría a los agentes a modificar sus comportamientos, a fin de adecuarse a la nueva realidad. En consecuencia, los modelos econométricos no podrían utilizarse para fines de formulación de políticas económicas. La crítica de Lucas desafió rigurosamente el modo en que el modelado econométrico se utilizaba tradicionalmente como instrumento para la evaluación de políticas económicas. Aunque la importancia empírica de la crítica sigue siendo motivo de debate, ella contribuyó a la imposición de nuevos patrones en el modelado de la interacción entre las reglas de política y las respuestas de los agentes privados.

El objetivo del presente trabajo es analizar los principales factores determinantes de la inversión privada en Brasil durante el período 1956-1996, mediante un instrumental moderno que incluye los tests de estacionariedad, cointegración y exogeneidad y que 
permite obviar la crítica de Lucas. Se emplea un modelo econométrico basado en los estudios más recientes para los países en desarrollo que considera, además de las variables más comunes, la influencia de las restricciones externas sobre la inversión privada. Por lo tanto, tiene un carácter más genérico que el de los modelos utilizados en estudios anteriores sobre Brasil. La meta principal es obtener un modelo para la inversión privada bien especificado y consistente con la teoría.

Los análisis de estacionariedad y cointegración permiten distinguir entre los efectos de corto y largo plazo de las variables explicativas. A su vez, los tests de exogeneidad comprueban la eficiencia de estimación del modelo y también aportan datos para promover políticas que incentiven la inversión del sector privado. Los resultados obtenidos indicaron que, den- tro del período en estudio, en la inversión privada influyeron positivamente el nivel del producto, la inversión pública y los créditos financieros, y negativamente el tipo de cambio y las condiciones de incertidumbre. Los tests de exogeneidad mostraron la importancia de la inversión pública y de los créditos financieros como instrumentos de una política que incentive la inversión privada.

El presente artículo consta de cinco secciones, siendo la primera esta introducción. La segunda hace algunas consideraciones sobre las variables utilizadas en este análisis y las fuentes de datos estadísticos. La tercera describe sucintamente la metodología que va a emplearse. En la cuarta se analizan los resultados empíricos obtenidos, y la quinta sección se destina a las conclusiones.

\section{II}

\section{Variables y datos estadísticos}

Según Servén y Solimano (1992), hay consideraciones teóricas y empíricas que sugieren que las variables relevantes para determinar la inversión privada en los países emergentes son: el producto interno, la tasa de interés real, la inversión pública, el crédito disponible para la inversión, la magnitud de la deuda externa, el tipo de cambio y la estabilidad macroeconómica. Como punto de partida de un análisis empírico, creemos que ésta es una descripción satisfactoria del problema.

Los datos de la inversión privada se obtuvieron de los de la formación bruta de capital fijo del sector privado, divulgados en el sistema de cuentas nacionales del Instituto Brasileño de Geografía y Estadística (IBGE). Comprenden, además de las inversiones privadas en construcción, máquinas y equipos. ${ }^{1}$

Con arreglo a la teoría neoclásica de la inversión, que tuvo su origen en el trabajo de Jogerson (1963), el valor del acervo de capital deseado por una empresa competitiva es una función positiva de su nivel de producto, pudiendo considerarse este último una aproximación del nivel de demanda. Si este resultado se extiende a niveles más agregados se consideraría el

\footnotetext{
${ }^{1}$ Algunos trabajos empíricos sobre Brasil incluyeron las inversiones de las empresas estatales como parte de la inversión pública. Considerando que lo importante es la característica de la inversión y no su propiedad, decidimos no seguir este predicamento.
}

producto de un país como una medida del nivel de demanda de todo el sector privado. En este estudio hemos utilizado los datos sobre el producto interno bruto (PIB) del sistema de cuentas nacionales del IBGE.

Otra variable que la teoría neoclásica considera relevante en las decisiones de inversión es la tasa de interés real, que en este caso representaría el costo de uso del capital o el costo del crédito para la empresa. Dado que un aumento de los intereses contribuye a desincentivar la inversión, cabría esperar una relación negativa entre las dos variables. Sin embargo, algunos estudios recientes (Agosin, 1994, sobre un conjunto de países en desarrollo; Cruz y Texeira, 1999, sobre Brasil) obtuvieron una relación no significativa desde el punto de vista estadístico, lo que parece evidenciar el carácter de corto plazo de las tasas de interés y las imperfecciones del mercado crediticio en los países en desarrollo. Para este análisis se utilizan los datos de la tasa de interés Over/Selic en el período 1973-1996, que representan el promedio de las tasas de interés de los títulos federales y son publicadas por el Banco Central del Brasil. Para el período 1956-1973, la serie se complementó con los datos de Ronci (1987).

En general, los gobiernos de los países en desarrollo tienen una participación considerable en las actividades económicas de éstos, lo que se justificaría por la ausencia del sector privado en grandes proyectos de 
inversión. La presencia del capital público afecta la inversión del sector privado de dos maneras distintas. Por un lado, el sector público compite con el privado por la apropiación de recursos escasos, tanto físicos como financieros, o incluso produciendo bienes comercializables que compiten con los productos privados. Esto se conoce en la literatura económica como efecto de desplazamiento (crowding-out). Por otro lado, el capital público puede aumentar la productividad mediante la generación de una externalidad positiva, como acontece en el caso de las inversiones en infraestructura y la provisión de bienes públicos, o incluso con una actuación anticíclica, elevando la demanda de insumos y servicios del sector privado. Este efecto positivo se denomina de atracción (crowding-in). Los datos utilizados para la inversión pública se obtuvieron de los de la formación bruta de capital fijo de la administración pública, divulgados en el sistema de cuentas nacionales del IBGE.

En los países emergentes, gran parte de las empresas tropiezan con restricciones en el mercado crediticio, lo que obedecería a la asimetría de información entre prestamistas y prestatarios y a una cierta fragilidad del mercado de capitales y de las intermediaciones financieras. En general, puede decirse que algunos sectores de los mercados de capital emergentes, como los financiamientos de largo plazo y el mercado de futuros, son poco desarrollados, lo que limita el financiamiento de la inversión privada al uso de créditos bancarios y a los empréstitos externos. Al disponerse de recursos de ese tipo se viabiliza la decisión de invertir cuando los fondos propios de los inversionistas son insuficientes para financiar sus proyectos. En este análisis se utilizaron los datos de desembolsos del BNDES, que representan los empréstitos de largo plazo con bajas tasas de interés destinados a financiar adquisiciones de bienes de capital.

La magnitud de la deuda externa es una de las variables que representa la influencia de las restricciones del crédito externo en el financiamiento de las actividades productivas de los países emergentes. Según Servén y Solimano (1992), las bajas tasas de inversión durante los años ochenta reflejaron la caída de la transferencia de recursos externos a los países altamente endeudados. Además, los valores elevados de la deuda exigieron una transferencia de recursos, que antes se destinaban al financiamiento de empresas locales, para pagar los cargos y servicios en el exterior. Los datos utilizados aquí se refieren a los de la relación deuda externa/PIB, publicados por el Banco Central del Brasil.
Dado que representa uno de los componentes que determinan el costo real de las importaciones, el tipo de cambio puede influir en el nivel de la inversión privada. Una desvalorización cambiaria aumenta los costos reales de adquisición de los bienes de capital importados, con lo cual se reduce la rentabilidad del sector privado y la inversión puede declinar. Además, una desvalorización cambiaria en términos reales puede provocar una reducción del ingreso real de la economía en su conjunto, disminuyendo también los niveles de actividad y capacidad productiva deseados por las empresas.

Por otro lado, la desvalorización del tipo de cambio real puede tener un impacto positivo en la inversión de los sectores que producen bienes transables con el exterior, pues aumenta la competitividad y el volumen de las exportaciones. Los datos utilizados son los del tipo de cambio nominal en relación con el dólar (promedio del período, venta) informado por el Banco Central del Brasil. ${ }^{2}$

De acuerdo con la teoría de la irreversibilidad de la inversión (Pindyck,1988), los gastos en capital fijo no pueden recuperarse completamente si la empresa decide venderlo en una fecha futura. El hecho de que muchos bienes de capital sean específicos de la empresa y tengan un valor de reventa menor que su precio de compra transforma la inversión en un costo irrecuperable. En consecuencia, el capital instalado no podrá utilizarse para otros fines sin que la empresa deba incurrir en costos.

Según Caballero (1993), la inversión es irreversible principalmente para los países en desarrollo, donde el mercado secundario de bienes de capital no es perfecto y hay varios tipos de costos de ajuste. Si se admite dicha hipótesis, la presencia de incertidumbres puede tener una gran influencia en la decisión de invertir, pues si el futuro es incierto, cualquier aumento de la capacidad productiva presente puede dejar a la empresa con un exceso de capital, que no se podrá eliminar sin costos. Eso explicaría por qué las empresas se muestran renuentes a realizar grandes inversiones, incluso durante períodos auspiciosos. En este sentido, la estabilidad económica y la credibilidad de las políticas públicas desempeñarían un papel importante en estimular la inversión. Para los fines del presente

\footnotetext{
2 Si bien Brasil tiene un patrón de comercio exterior bastante diversificado, con varios países de destino y origen, creemos que las variaciones del tipo de cambio en relación con el precio del dólar expresan, en promedio, las variaciones de un tipo de cambio efectivo.
} 
trabajo, la variación de la tasa de inflación se utiliza como una aproximación para evaluar las condiciones de incertidumbre en la economía. Los datos utilizados se refieren a la variación de la tasa de inflación calculada conforme al índice general de precios-disponibilidad interna (IGP-DI) que entrega la Fundación Getulio Vargas.

La mayoría de los datos que van a utilizarse en las variables explicativas sólo están disponibles con una periodicidad anual. Es el caso, por ejemplo, de los

\section{III}

\section{Metodología}

Los procedimientos econométricos se realizarán en cuatro etapas. En la primera se determinará el orden de integración de cada una de las series utilizadas en el análisis, mediante la aplicación de los tests de estacionariedad o de raíces unitarias. Al comienzo, se efectuará el test de Dickey-Fuller aumentado (Dickey y Fuller, 1981), denotado por la sigla DFA. La decisión sobre la estacionariedad de una serie cronológica se basará también en la inspección visual de su correlograma, dado que los tests de raíces unitarias son una formalización de esta inspección.

Habida cuenta de que cuando hay quiebres estructurales el test DFA puede señalar erróneamente la no estacionariedad de una serie que sea estacionaria, se efectuará el test de raíces unitarias sugerido por Perron. ${ }^{3}$ Este test procura establecer el orden de integración de una serie cronológica tomando en cuenta la posibilidad de que ocurran cambios estructurales en su comportamiento.

En la segunda etapa se identificarán las variables y sus desfases respectivos, que son significativos en la ecuación de la inversión privada. Siguiendo la metodología "de lo general a lo particular", se estimará primero un modelo general con desfases distribuidos, conocido como ADL. ${ }^{4}$ Mediante los tests de restricciones, el modelo es reducido gradualmente a través de la eliminación de las variables y desfases que se muestren estadísticamente no significativos.

\footnotetext{
${ }^{3}$ El test que se utilizará se denomina additive outlier. Para mayores detalles, véase Perron (1989).

${ }^{4}$ Véase más sobre las bases de esta metodología en Charemza y Deadman (1997).
}

datos sobre la inversión pública y privada, el crédito y la deuda externa. Como vamos a analizar un número razonable de variables explicativas, además de modelos que incluyen desfases para cada variable, tratamos de obtener una muestra con el mayor número posible de observaciones. Además, durante el período 19561996 la economía brasileña atravesó por diversos regímenes, lo que torna la investigación de la crítica de Lucas en un tema interesante para este estudio empírico.

En la tercera etapa se utilizará el método de Engle y Granger (1987) para verificar la hipótesis de cointegración en las series que se muestren integradas de orden uno, con la estimación ulterior del modelo de diferencias con el mecanismo de corrección de errores. También se utilizará el método de Johansen (1988), que analiza los vectores de cointegración mediante un modelo VAR, para determinar con mayor precisión el número de relaciones de cointegración y las estimaciones de los vectores de coeficientes de estas relaciones. Esta etapa se justifica porque la tendencia de una serie cronológica puede ser de dos tipos: determinista o estocástica. En el primer caso, la serie puede transformarse en estacionaria mediante la inclusión de la variable tiempo en un modelo de regresión. En cambio, con una tendencia estocástica se necesitan tests en cuanto a la cointegración para verificar si, incluso siendo individualmente no estacionaria, una combinación lineal de dos o más series cronológicas puede generar un residuo estacionario. La cointegración de dos o más series cronológicas sugiere una relación de largo plazo entre ellas, mientras que el mecanismo de corrección de errores sólo indica la inclusión del residuo estacionario desfasado en el modelo de corto plazo, para conciliar el comportamiento de corto plazo con el equilibrio de largo plazo.

En la cuarta etapa se realizarán los tests de exogeneidad débil y superexogeneidad. Según Engle, Hendry y Richard (1983), la hipótesis de exogeneidad débil asegura la realización de inferencias eficientes de los parámetros cuando nos restringimos al análisis del modelo condicional. En el presente estudio, este modelo tiene a la inversión privada como variable endógena. 
Se realizarán los tests de exogeneidad débil respecto a los parámetros de las ecuaciones de corto y largo plazo.

La combinación de exogeneidad débil e invarianza estructural de los parámetros estimados lleva al concepto de superexogeneidad. Si una variable explicativa fuera superexógena, las alteraciones en su distribución no tendrían efectos sobre los parámetros del modelo condicional. Siendo así, sus efectos sobre la variable endógena pueden analizarse en términos de simulaciones de políticas y mediante la realización de inferencias en ambientes sujetos a intervenciones. De este modo, la verificación de superexogeneidad refuta la crítica de Lucas que, en síntesis, cuestiona el uso de los parámetros estimados de un modelo econométrico para efectuar simulaciones de política, ya que los agentes están constantemente revisando sus expectativas ante los cambios del entorno económico.

\section{IV}

\section{Análisis de los resultados econométricos}

En todo el análisis econométrico efectuado se utilizó el logaritmo natural de las series cronológicas de cada variable. Esto obedece a que las series expresadas en logaritmos presentan varianzas aproximadamente constantes, mientras que la varianza de una serie de nivel tiende a aumentar con el tamaño de la muestra. Tal como en Greene y Villanueva (1995) y Rocha y Teixeira (1996), la variable tasa de interés real se definió como: $(1+\mathrm{i} / 100)$, donde $i$ es la tasa de interés

$$
(1+\pi / 100)
$$

nominal en términos porcentuales y $\pi$ es la inflación porcentual. $^{5}$

\section{Tests de estacionariedad}

El análisis visual de los gráficos y correlogramas de las series en nivel y en primeras diferencias indica la posibilidad de que las series variación de la tasa de inflación y tasa de interés real sean estacionarias, mientras que las demás series parecen ser integradas de orden uno. ${ }^{6}$

a) Resultados del test de Dickey-Fuller aumentado Los resultados del test de Dickey-Fuller aumentado para las series en nivel y en primeras diferencias

\footnotetext{
${ }^{5}$ Los valores de las series de inversión privada, producto, inversión pública, tipo de cambio y crédito están expresados en millones de reales de 1995. Para las series de inversión, utilizamos el deflactor de la formación bruta de capital fijo del IBGE. Para las demás series utilizamos el índice de precios-disponibilidad interna (IGP-DI). Todos los resultados econométricos se obtuvieron con los programas computacionales PC-GIVE y PC-FIML, versión 9.10.

${ }^{6}$ Por razones de espacio, omitimos la presentación de gráficos y correlogramas.
}

figuran en el cuadro 1. En la segunda columna están los parámetros determinísticos (constante y tendencia lineal) que presentaron un valor $\mathrm{t}$ significativo a nivel de $10 \% \mathrm{y}$, por ello, se incluyeron en la regresión de cada una de las variables. La tercera columna muestra el número de desfases introducidos en cada regresión para eliminar una posible autocorrelación de los residuos, los que se determinaron mediante la minimización de los criterios de Akaike y Schwartz. La última columna presenta el valor de la estadística $\tau$-DFA, o para el caso en que ningún desfase se haya mostrado significante, de la estadística $\tau$-DF.

Los resultados obtenidos muestran que las series tasa de interés y variación de la tasa de inflación son integradas de orden cero o estacionarias, mientras que las primeras diferencias de las series de inversión privada, inversión pública, deuda externa, tipo de cambio y crédito rechazan la hipótesis nula de no estacionariedad y son, por lo tanto, integradas de orden uno. De acuerdo con el análisis gráfico hecho previamente, la serie del PIB fue la única que presentó un resultado diferente del esperado. Con miras a obtener un resultado más confiable de la serie mencionada, tendremos que recurrir al test de Perron.

\section{b) Resultados del test de Perron}

El test de Perron se realizó para todas las series que se revelaron no estacionarias con el test DFA, con el fin de verificar si ellas son realmente no estacionarias o están sujetas a un quiebre estructural que origina un cambio permanente de sus promedios. ${ }^{7}$ El cua-

\footnotetext{
${ }^{7}$ Para la determinación precisa del período de ocurrencia del quiebre estructural en cada serie (o del quiebre principal, en el caso de haber varios), se procedió a analizar los gráficos recursivos de las
} 
Resultados del test ADF: series en nivel y en primera diferencia

\begin{tabular}{|c|c|c|c|}
\hline Variable & Parámetros determinísticos & Desfases & $\tau$-DFA o $\tau$-DF \\
\hline Log inv. privada & Constante & 1 & -1.756 \\
\hline Log producto & Constante y tendencia & 4 & -2.406 \\
\hline Log tasa de interés & Constante y tendencia & 0 & $-4.29^{\mathrm{a}}$ \\
\hline Log inv. pública & Constante & 1 & -1.864 \\
\hline Log deuda externa & & 3 & -0.619 \\
\hline Log tipo de cambio & Constante y tendencia & 4 & -2.955 \\
\hline$\Delta$ Log inflación & & 6 & $-2.230^{\mathrm{b}}$ \\
\hline$\Delta \log$ inv. privada & Constante & 1 & $-4.742^{b}$ \\
\hline$\Delta$ Log producto & & 1 & -1.603 \\
\hline$\Delta$ Log inv. pública & & 0 & $-5.765^{\mathrm{a}}$ \\
\hline$\Delta \log$ deuda externa & & 2 & $-3.959^{a}$ \\
\hline$\Delta$ Log tipo de cambio & Constante & 4 & $-3.183^{b}$ \\
\hline$\Delta \log$ crédito & & 3 & $-2.039^{b}$ \\
\hline
\end{tabular}

a Denota significación a nivel de $1 \%$.

b Denota significación a nivel de $5 \%$.

CUADRO 2

Test de Perron para las series en nivel y en primeras diferencias

\begin{tabular}{lclc}
\hline Variable & $\mathrm{t}$ & Variable & $\mathrm{t}$ \\
\hline Log inv. privada & -0.839 & $\Delta \log$ inv. privada & $-5.396^{\mathrm{a}}$ \\
Log producto & -1.202 & $\Delta \log$ producto & $-3.835^{\mathrm{b}}$ \\
Log inv. pública & -2.528 & $\Delta \log$ inv. pública & $-6.484^{\mathrm{a}}$ \\
Log deuda externa & -1.639 & $\Delta \log$ deuda externa & $-8.608^{\mathrm{a}}$ \\
Log tipo de cambio & -0.236 & $\Delta \log$ tipo de cambio & $-7.037^{\mathrm{a}}$ \\
Log crédito & -2.080 & $\Delta \log$ crédito & $-7.486^{\mathrm{a}}$ \\
\hline
\end{tabular}

a Indica rechazo de la hipótesis nula al nivel de significación de $1 \%$.

b Indica rechazo de la hipótesis nula al nivel de significación de $5 \%$.

dro 2 presenta los resultados del test de Perron para las series en nivel y en primeras diferencias. En la segunda y cuarta columnas figuran los valores obtenidos para la estadística t. Los valores críticos superiores de t, proporcionados por Charemza y Deadman (1997, pp. 301-303), son -3.48 y -4.15 a los niveles de significación de $5 \%$ y $10 \%$, respectivamente.

La serie del PIB se mostró estacionaria en primeras diferencias, confirmando el resultado obtenido por el análisis de su gráfico y correlograma. Por tanto, el resultado del test DFA para la serie mencionada parece estar sesgado, debido a la presencia de un quiebre es-

estimaciones de cada serie, en un modelo sólo con constante y tendencia lineal. Salvo la inversión privada y el tipo de cambio, las series presentaron mayores cambios estructurales a comienzos de los años ochenta, período marcado por un bajo crecimiento económico, inflación elevada y la crisis de la deuda. tructural. Para las demás series, los resultados confirman los obtenidos por el test DFA y el análisis de los correlogramas.

Tras la realización de los tests de raíces unitarias, podemos afirmar que dentro del período considerado:

i) las series de inversión privada, producto, inversión pública, deuda externa, tipo de cambio y crédito son integradas de orden uno I(1), siendo no estacionarias en nivel y estacionarias en las primeras diferencias.

ii) las series de la tasa de interés y variación de la tasa de inflación son estacionarias en nivel ó $\mathrm{I}(0)$.

\section{Tests de restricciones sobre variables y des- fases}

La metodología utilizada fue la de comenzar por la estimación de un modelo general y, aplicando tests de restricciones, reducir gradualmente su tamaño mediante la eliminación de los desfases y variables que se mostraron no significativos.

El caso general se describirá como un modelo de desfases distribuidos, conocido como ADL. Este modelo tiene a la inversión privada como variable dependiente, la que se expresa como una función de sus propios desfases y de los valores corrientes y desfasados de las demás variables cuyas series se señalaron como I(1): producto, inversión pública, deuda externa, tipo de cambio y crédito.

Debido a la gran cantidad de variables explicativas y el número relativamente pequeño de observaciones, se inició el análisis con la estimación de un 
modelo ADL (3), con tres desfases de cada variable. No obstante, se comprobó una fuerte correlación de la serie de la inversión pública con las series del PIB, el crédito y el tipo de cambio. Con el fin de no incurrir en el problema de multicolinealidad, decidimos estimar por separado dos modelos ADL para la inversión privada: el primero tiene como variables dependientes el PIB, la deuda, el tipo de cambio y el crédito, mientras que el segundo sólo tiene la inversión pública como variable explicativa. $^{8}$

Los resultados de los tests LM, de significación conjunta para el primer modelo, indicaron que la contribución de los tres desfases es significativa al nivel de $10 \%$. Además, los tests Lm para cada variable muestran que la deuda externa no es significativa en la determinación de la inversión privada, dentro del período considerado. Así, el primer paso para reducir el modelo es la eliminación de las variables que se revelaron no significativas. El cuadro 3 presenta los valores de la suma de los cuadrados de los residuos (SCR), de la desviación estándar estimada de los residuos $(\sigma)$ y del criterio de Schwarz para los modelos ADL con y sin la presencia de la variable deuda externa.

Los valores mencionados no mostraron grandes diferencias entre los dos modelos. El resultado del test F muestra el no rechazo de la hipótesis de que todos los coeficientes de la variable deuda externa son iguales a cero, confirmando la posibilidad de exclusión de los mismos. El análisis de los gráficos recursivos del modelo permitió verificar además la existencia de un quiebre estructural en 1995. En el modelo se incluyó una variable de impulso ficticia correspondiente a ese año.

En el segundo modelo, los tests de significación conjunta para cada desfase muestran que el segundo y tercer desfase de la inversión privada y pública no son

CUADRO 3

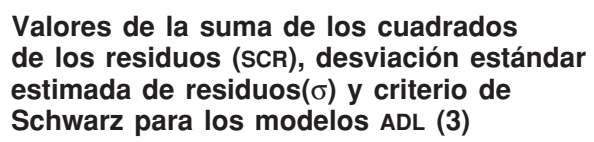

\begin{tabular}{lccc}
\hline Modelo & SCR & $\sigma$ & Schwarz \\
\hline ADL (3) & 0.1008 & 0.0728 & -4.1134 \\
ADL (3) s/var. deuda. & 0.1341 & 0.0764 & -4.2109 \\
\hline
\end{tabular}

Modelo ADL (3) $\rightarrow$ ADL (3) s/var. deuda ext. : F(4,19) = 1.5691 [0.2231]

\footnotetext{
${ }^{8}$ El test de especificación de Ramsey se aplicó en cada modelo ADL y no detectó errores de especificación.
}

CUADRO 4

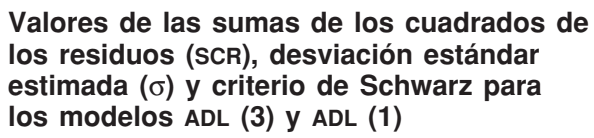

\begin{tabular}{lccc}
\hline Modelo & SCR & $\sigma$ & Schwarz \\
\hline ADL (3) & 0.4372 & 0.1188 & -3.7948 \\
ADL (1) & 0.5068 & 0.1203 & -4.0300 \\
\hline
\end{tabular}

Modelo ADL (3) $\rightarrow$ ADL (1) $:$ F(4,31) = 1.233 [0.3172]

significativos en el análisis. El cuadro 4 presenta los valores de la suma de los cuadrados de los residuos (SCR), de la desviación estándar estimada de los residuos $(\sigma)$ y del criterio de Schwarz para los modelos $\operatorname{ADL}(3)$ y $\operatorname{ADL}(1)$, además del test $\mathrm{F}$ de reducción de parámetros.

Los valores no mostraron grandes diferencias entre los dos modelos. El resultado del test F muestra el no rechazo de la hipótesis de que los coeficientes del segundo y tercer desfase son conjuntamente iguales a cero, confirmando la posibilidad de excluirlos. El análisis de los gráficos recursivos del modelo permitió verificar además la existencia de un quiebre estructural en 1990.

\section{Tests de cointegración}

Para los análisis de cointegración, sólo se utilizaron las variables integradas de orden uno que se revelaron estadísticamente significativas en la determinación de la inversión privada: producto, tipo de cambio y crédito, para el primer modelo, e inversión pública en el segundo modelo.

\section{a) Resultados obtenidos con el método de Engle- Granger}

Los coeficientes de la relación de largo plazo entre la inversión privada y las variables significativas se obtienen a partir de los modelos específicos de la sección anterior. Las ecuaciones de largo plazo estimadas presentaron los siguientes resultados (ver Modelo 1 y 2).

Los DFA de los residuos de las ecuaciones [4.1] y [4.2] indican estacionariedad de nivel. Como las series de inversión privada, producto, inversión pública, tipo de cambio y crédito son todas I(1), tenemos dos relaciones de equilibrio de largo plazo dadas por [4.1] y [4.2].

En la primera ecuación estimada, dentro del período 1959-1996, los coeficientes del producto y el crédito tienen signo positivo, mientras que los coefi- 
Modelo 1 [4.1]

Log inv. privada $=0.7509$ Log producto $-0.2312 \log$ tipo de cambio +0.1702 Log crédito -0.2424 i1995

$\begin{array}{lllll}\text { (estadística t) } \quad(70.2432) & (-9.8593) & (10.6842)\end{array}$

Test de Wald de significación conjunta: $\operatorname{Chi}^{2}(4)=2.3624 \mathrm{e}+005[0.0000]^{* *}$

Test DFA de residuo: $\tau$-DFA $=-2.67 * *$

Modelo 2 [4.2]

Log inv. privada $=1.2120$ Log inv. pública -1.9550 i1990

(estadística t) (64.0931) (-1.9492)

Test de Wald de significación conjunta : $\operatorname{Chi}^{2}(4)=6967.7[0.0000]^{* *}$

Test DFA de residuo: $\tau$-DF $A=-2.126^{*}$

* Indica rechazo de la hipótesis nula al nivel de significación de 5\%.

** Indica rechazo de la hipótesis nula al nivel de significación de $1 \%$.

cientes del tipo de cambio y de la variable ficticia son negativos. Los coeficientes positivos del producto y del crédito muestran que la inversión privada fue estimulada tanto por el nivel de actividad de la economía como por la disponibilidad de financiamiento de largo plazo, lo que concuerda con la mayoría de los resultados empíricos hallados en la literatura. El coeficiente negativo del tipo de cambio muestra que, en el largo plazo, la desvalorización/depreciación cambiaria provocó una caída de la inversión. Probablemente, debido a la reducción del ingreso real de la economía y el aumento del costo de los bienes de capital importados, lo que lleva a la reducción del nivel de actividad como un todo.

La segunda ecuación estimada, dentro del período 1957-1996, muestra el predominio del efecto inducción, donde las inversiones en bienes públicos presentaron un impacto positivo sobre la inversión del sector privado. Observamos, además, de acuerdo con el signo negativo de los coeficientes de las variables de impulso ficticias en [4.1] y [4.2], una caída de los niveles de la inversión privada en los años noventa.

Una vez analizada la dinámica de largo plazo de la inversión privada, el paso siguiente es determinar las relaciones de corto plazo entre las variables. Estas relaciones están representadas por los modelos con las primeras diferencias de las variables I(1), con la inclusión del mecanismo de corrección de errores (MCE) y de las variables tasa de interés real y variación de la tasa de inflación, cuyas series se mostraron estacionarias en nivel.

Los cuadros 5 y 6 presentan los resultados de las estimaciones, junto con los tests de diagnóstico del residuo. En los dos modelos estimados, el término mecanismo de corrección de errores presentó un coeficiente negativo y significativo, lo que confirma, conforme al teorema de la representación de Granger,
CUADRO 5

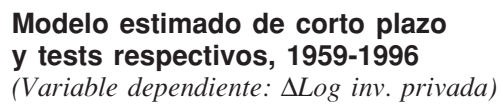

\begin{tabular}{|c|c|c|c|c|}
\hline Variable & Coeficiente & $\begin{array}{c}\text { Desviación } \\
\text { estándar }\end{array}$ & $\mathrm{t}$ & t prob. \\
\hline$\Delta \log$ inv. privada -1 & 0.4918 & 0.1224 & 4.018 & 0.0004 \\
\hline$\Delta \log$ producto & 1.4152 & 0.2679 & 5.283 & 0.0000 \\
\hline$\Delta$ Log tipo de cambio & -0.4274 & 0.0881 & -4.851 & 0.0000 \\
\hline$\Delta \log$ tipo de cambio -2 & -0.1085 & 0.0613 & -1.772 & 0.0865 \\
\hline$\Delta$ Log crédito & 0.1619 & 0.0357 & 4.542 & 0.0001 \\
\hline$\Delta \log$ inflación & -0.0598 & 0.0314 & -1.906 & 0.0602 \\
\hline MCE 1 & -0.7756 & 0.1389 & -5.585 & 0.0000 \\
\hline i1995 & -0.3314 & 0.1471 & -2.253 & 0.0317 \\
\hline \multirow[t]{2}{*}{$R^{2}-0.7649$} & \multicolumn{4}{|c|}{$\sigma=0.0775 \quad$ D.W $=2.24$} \\
\hline & \multicolumn{4}{|c|}{ Tests de residuos } \\
\hline Test LM & Estadístic & & t prob. & \\
\hline (autocorrelación) & $\mathrm{F}(2,28)=1$. & 0856 & 0.3515 & \\
\hline ARCH 1 & $\mathrm{~F}(1,28)=1$. & 0029 & 0.3252 & \\
\hline Normalidad & $X^{2(2)}=3$. & 3579 & 0.1866 & \\
\hline White & $\mathrm{F}(15,14)=0$. & 2636 & 0.9925 & \\
\hline & \multirow{2}{*}{\multicolumn{4}{|c|}{ Test de especificación de la regresión }} \\
\hline Ramsey & $F(1,29)=0$. & & & \\
\hline
\end{tabular}

que las series se cointegran. Los resultados de los tests del residuo indican ausencia de autocorrelación, conforme al test LM, y de heterocedasticidad, conforme a los tests ARCH1 y de White. Los residuos se mostraron normales, de acuerdo con el valor estadístico $\mathrm{X}^{2}$. Los tests de Ramsey revelan que las dos regresiones están bien especificadas.

En el primer modelo, estimado para el período 1959-1996, las primeras diferencias de las series del producto y el crédito presentaron coeficientes positivos, revelándose como factores importantes de la inversión privada también en el corto plazo. Los coeficientes negativos del tipo de cambio, en primeras diferencias, 
sin desfase y con dos desfases, indican que la caída de las inversiones debida a la desvalorización/depreciación cambiaria ocurrió tanto en el largo como en el corto plazo. Las variaciones de la tasa de inflación también se revelaron significativas en la determinación de la inversión privada, lo que indica que las condiciones de incertidumbre en la economía contribuyeron a reducir el nivel de la inversión. Los coeficientes de la tasa de interés no se mostraron estadísticamente significativos, lo que indica que sus variaciones en el corto plazo no llegaron a afectar la inversión de manera significativa.

\begin{tabular}{|c|c|c|c|c|}
\hline \multirow{2}{*}{ Variable } & \multicolumn{4}{|c|}{$\begin{array}{l}\text { Modelo estimado de corto plazo y } \\
\text { los tests respectivos, 1958-1996 } \\
\text { (Variable dependiente: } \Delta \log \text { inv. privada) }\end{array}$} \\
\hline & Coeficiente & $\begin{array}{c}\text { Desviación } \\
\text { estándar }\end{array}$ & $\mathrm{t}$ & t prob. \\
\hline$\Delta \log$ inv. pública -1 & 0.2177 & 0.1164 & 1.871 & 0.0695 \\
\hline MCE 2 & -0.1323 & 0.0329 & -4.011 & 0.0003 \\
\hline i1990 & -0.3104 & 0.1020 & -3.043 & 0.0044 \\
\hline \multirow[t]{2}{*}{$R^{2}=0.5422$} & \multicolumn{4}{|c|}{$\sigma=0.1018 \quad$ D.W $=1.85$} \\
\hline & \multicolumn{4}{|c|}{ Tests del residuo } \\
\hline Test & \multirow{2}{*}{\multicolumn{2}{|c|}{$\begin{array}{l}\text { Estadistica } \\
\mathrm{F}(2.34)=2.0811\end{array}$}} & \multirow{2}{*}{\multicolumn{2}{|c|}{$\begin{array}{l}\mathrm{t} \text { prob. } \\
0.1404\end{array}$}} \\
\hline LM (autocorrelación) & & & & \\
\hline ARCH 1 & \multicolumn{2}{|c|}{$F(1,34)=2.3777$} & \multicolumn{2}{|l|}{$\begin{array}{l}0.1404 \\
0.1323\end{array}$} \\
\hline Normalidad & \multicolumn{2}{|c|}{$X^{2}(2)=4.246$} & \multicolumn{2}{|l|}{0.1197} \\
\hline $\begin{array}{l}\text { White } \\
\text { (heterocedestacidad) }\end{array}$ & \multicolumn{2}{|c|}{$\mathrm{F}(5,30)=0.6392$} & 0.6716 & \\
\hline \multirow[b]{2}{*}{ Ramsey } & \multicolumn{4}{|c|}{ Test de especificación de la regresión } \\
\hline & $\mathrm{F}(1,35)=1$ & 6402 & 0.2087 & \\
\hline
\end{tabular}

El coeficiente estimado para la variable de impulso ficticia apunta nuevamente a una caída en 1995.

El segundo modelo estimado muestra el impacto positivo de la inversión pública desfasada en un período, además de la caída de la inversión privada en 1990. El resultado obtenido confirma el efecto inducción: la maduración de las inversiones públicas influyó de modo positivo en el capital privado, aumentando su productividad, lo que concuerda con los resultados de Cruz y Teixeira (1999) para el largo plazo.

\section{b) Resultados obtenidos con el método de Johansen}

Dado que se utiliza el modelo var, donde no se especifican a priori las variables endógenas y exógenas, y admitiendo también la posibilidad de que exista más de un vector de cointegración, el análisis de cointegración con el método de Johansen es de carácter más general que el método de Engle-Granger.

Considerando los resultados obtenidos en la sección IV.2, se estimó un VAR con tres desfases para las variables inversión privada, producto, tipo de cambio y crédito, y otro modelo VAR con un desfase para las variables inversión privada e inversión pública. Los cuadros 7 y 8 muestran los resultados de la aplicación del procedimiento de Johansen a partir de los modelos, VAR (3) y VAR (1), respectivamente. ${ }^{9}$

En ambos modelos, los resultados de los valores

\footnotetext{
${ }^{9}$ Aquí empleamos un procedimiento semejante al de la sección IV.2 indicada para analizar la significación estadística de cada variable y cada desfase de los modelos var.
}

CUADRO 7

Análisis de cointegración con el método de Johansen: VAR (3)

\begin{tabular}{|c|c|c|c|c|}
\hline Hipótesis & $r=0$ & $r \leq 1$ & $r \leq 2$ & $r \leq 3$ \\
\hline$\mu_{\text {mínime }}$ & $39.29^{\mathrm{a}}$ & 14.01 & 4.215 & 0.599 \\
\hline Val. crítico a $5 \%$ & 27.1 & 21.0 & 14.1 & 3.8 \\
\hline Hipótesis & $r=0$ & $r=1$ & $r=2$ & $r=3$ \\
\hline$\mu_{\max }$ & $58.11^{\mathrm{a}}$ & 18.82 & 4.814 & 0.599 \\
\hline \multirow[t]{7}{*}{ Val. crítico a $5 \%$} & 47.2 & 29.7 & 15.4 & 3.8 \\
\hline & & Autovectores $\beta$, & & \\
\hline & Log inv. privada & Log producto & Log tipo de cambio & Log crédito \\
\hline & 1.0000 & -0.7108 & 0.2501 & -0.1829 \\
\hline & 0.0121 & 1.0000 & 0.4045 & -0.2917 \\
\hline & -4.1359 & -11.0740 & 1.0000 & 10.3610 \\
\hline & -1.0034 & -2.1547 & -0.4419 & 1.0000 \\
\hline & & Coeficientes $\alpha$ & & \\
\hline Log inv. privada & -0.6334 & 0.8003 & 0.0011 & 0.0119 \\
\hline Log producto & 0.0733 & 0.0825 & -0.0003 & 0.0079 \\
\hline Log tipo de cambio & -0.0254 & -0.5612 & -0.0114 & -0.0033 \\
\hline Log crédito & 2.0316 & 2.1885 & -0.0115 & 0.0002 \\
\hline
\end{tabular}

a Indica rechazo de la hipótesis nula al nivel de significación de $1 \%$. 
CUADRO 8

Análisis de cointegración con el método de Johansen: vaR (1)

\begin{tabular}{lll}
\hline Hipótesis & $r=0$ & $r \leq 1$ \\
$\mu_{\text {mínimo }}$ & $19.28^{\mathrm{a}}$ & 3.112 \\
Val. crítico a 5\% & 14.1 & 3.8 \\
Hipótesis & $r=0$ & $r=1$ \\
$\mu_{\max }$ & $22.39^{\mathrm{a}}$ & 3.112 \\
Val. crítico a 5\% & 15.4 & 3.8
\end{tabular}

\begin{tabular}{lcc} 
& Autovectores $\beta^{\prime}$ & \\
& Log inv. privada & Log inv. pública \\
& 1.0000 & -1.9316 \\
& -1.4506 & 1.0000 \\
& & \\
Log inv. privada & -0.1034 & \\
Log inv. pública & 0.1797 & 0.0461 \\
\hline
\end{tabular}

a Indica rechazo de la hipótesis nula al nivel de significación de $1 \%$.

estadísticos mínimo y máximo apuntan al rechazo de la hipótesis nula de ausencia de cointegración y al no rechazo de la hipótesis, en el máximo, de un vector de cointegración. Por lo tanto, las estadísticas sugieren que existe sólo un vector de cointegración en los dos modelos estimados. La primera línea de la matriz $\beta$ ' aporta los coeficientes estimados para las ecuaciones de largo plazo que tienen a la inversión privada como variable endógena:

Log inv. priv. $=0.7108 \mathrm{Log}$ producto $-0.2501 \mathrm{Log}$ tipo de cambio +0.1829 Log crédito

Log inv. priv. $=1.9316$ Log inv. pública

Los resultados son semejantes a los obtenidos con el método de Engle-Granger. Los valores de los coeficientes de la ecuación [4.3] son aproximadamente iguales a los obtenidos en la ecuación [4.1]. El coeficiente obtenido en la ecuación [4.4] se mostró mayor que el coeficiente estimado en la ecuación [4.2].

\section{Tests de exogeneidad}

El primer test de exogeneidad que se realizó se refiere a los parámetros de las ecuaciones de largo plazo obtenidos en la sección IV.3.b. Sobre la base de los trabajos de Hendry y Mizon (1993) y Johansen (1994), la hipótesis de exogeneidad se formula como una restricción paramétrica en la matriz de ajuste $\alpha$. El cuadro 9 presenta los resultados obtenidos en cada uno de los modelos. Se calculó el test estadístico LR para cada una de las variables incluidas en los modelos VAR de la sección indicada. El valor crítico, a nivel de 5\% de significación, es 3.84.

Con arreglo a los resultados, rechazamos la hipótesis de que los coeficientes de ajuste $\alpha$ de las variables inversión privada y crédito del modelo VAR (3) son nulos. Lo mismo puede decirse de las variables inversión privada e inversión pública del modelo VAR (1). Por lo tanto, los procesos marginales generadores de estas variables contienen informaciones relevantes para las relaciones de cointegración. Por ello, no parece adecuado incluir el crédito y la inversión pública como variables exógenas en las ecuaciones de largo plazo, pues las estimaciones se tornaron ineficientes.

\section{a) Tests de exogeneidad débil para los parámetros del modelo de largo plazo}

El primer paso, antes de realizar estos tests, es la formulación de los modelos marginales para las variables explicativas presentes en los modelos de corto plazo. La tentativa de especificar los procesos marginales se inició con la estimación de un modelo ADL (3). Siguiendo la metodología "de lo general a lo particular", se eliminaron del modelo los términos no significativos. El cuadro 10 presenta los resultados de los modelos marginales obtenidos empíricamente.

Para que una variable sea considerada exógena débil en el modelo de corto plazo, deberá satisfacer las siguientes condiciones: i) su modelo marginal no contiene el término MCE de corrección de errores; ii) los

CUADRO 9

Tests de exogeneidad débil en relación con los parámetros de largo plazo

$\begin{array}{lc}\begin{array}{l}\text { Modelo VAR (3) } \\ \text { Variable } \\ \text { Estadística LR }\end{array} & \begin{array}{c}\text { Log inv. privada } \\ 4.3106^{\mathrm{a}}\end{array} \\ \begin{array}{l}\text { Modelo VAR (1) } \\ \text { Variable }\end{array} & \text { Log inv. privada } \\ \text { Estadística LR } & 4.7946^{\mathrm{a}}\end{array}$


CUADRO 10

\section{Modelos marginales estimados para las variables explicativas}

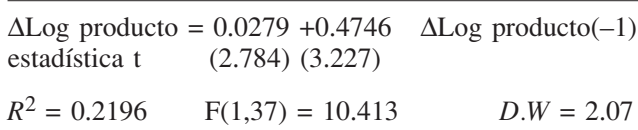

$\Delta \log$ tipo de cambio $=-0.0842-0.3780 \quad \Delta \log$ tipo de cambio(-3) +0.6059 i 94

estadística $\mathrm{t} \quad(-3.196)(-3.272)$

$R^{2}=0.6530 \quad \mathrm{~F}(2,34)=11.667 \quad$ D. $W=2.16$

$\Delta \log$ créd. $=-0.2345-0.4836 \quad \Delta \log$ créd. $(-1)-0.4887 \quad \Delta \log$ créd.(-2) $-0.3873 \quad \Delta \log$ créd. $(-3)+4.0646 \quad \Delta \log$ prod. $(-3)$

$\begin{array}{lllll}\text { estadística t } & (-2.055)(-2.862) & (-3.004) & (-2.164) & (2.639)\end{array}$

$R^{2}=0.3564 \quad \mathrm{~F}(4,32)=3.433 \quad D . W=1.83$

$\Delta \log$ inflación $=-0.4476 \quad \Delta \log$ inflación $(-3)-8.3259 \quad \Delta \log$ producto $(-2)+9.0803 \quad \Delta \log$ producto $(-3)$

estadística t (-1.981) (-2.484) (2.648)

$R^{2}=0.2115 \quad$ D. $W=1.86$

$\Delta$ Log inv. pública $=0.1042 \Delta \log$ crédito $(-2)+0.3565$ i 86

estadística t (2.669) (3.054)

$R^{2}=0.3054 \quad D . W=2.11$

CUADRO 11

Resultados de los tests de exogeneidad débil: parámetros de corto plazo

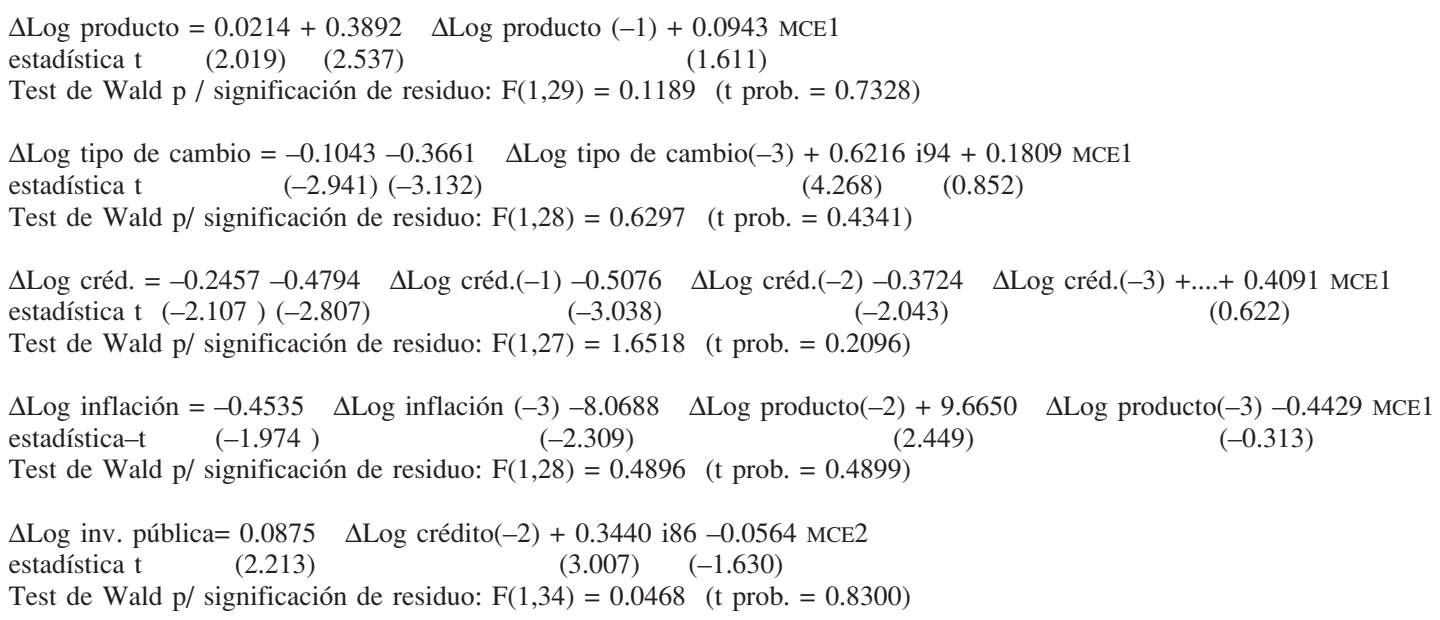

residuos de su modelo original no están correlacionados con los residuos del modelo de corto plazo.

El cuadro 11 presenta los resultados obtenidos para las estimaciones de los modelos marginales con la inclusión del término MCE. Se presentan también los resultados del test de Wald, a fin de que analicemos la significación de los residuos de cada uno de los modelos marginales en el respectivo modelo de corto plazo.

Conforme a los resultados de las estadísticas t para cada término MCE, y de las estadísticas F del test de Wald, todas las variables satisfacen las dos condiciones de exogeneidad débil. Por lo tanto, podemos concluir que las inferencias relativas a los parámetros de los dos modelos de corto plazo de la inversión privada pueden realizarse sin pérdida de información relevante.

b) Resultados de los tests de superexogeneidad

La manera más común de verificar la invarianza estructural de los parámetros de un modelo condicional consiste en verificar la significación del cuadrado de los residuos estimados en los modelos marginales dentro del propio modelo. Este tipo de test fue propues- 
Modelo marginal: $\Delta$ Log producto

Test LM p/ significación del cuadrado del residuo:

Modelo marginal: $\Delta$ Log tipo de cambio

Test LM p/ significación del cuadrado del residuo:

Modelo marginal: $\Delta$ Log créd.

Test LM p/ significación del cuadrado del residuo:

Modelo marginal: $\Delta$ Log inflación

Test LM p/ significación del cuadrado del residuo:

Modelo marginal: $\Delta$ Log inv. pública

Test LM p/ significación del cuadrado del residuo:
$\mathrm{F}(2,28)=1.1731$
(t prob. $=0.3241)$
$\mathrm{F}(2,26)=0.0283$
$(\mathrm{t}$ prob. $=0.9721)$
$\mathrm{F}(2,26)=0.8867$
(t prob. $=0.4241)$
$\mathrm{F}(2,26)=0.3661$
$(\mathrm{t}$ prob. $=0.6970)$
$\mathrm{F}(2,32)=0.5460$
$(\mathrm{t}$ prob. $=0.5846)$

to por Engle y Hendry (1993). Para aceptar la superexogeneidad, el cuadrado de los residuos no debe mejorar la estimación del modelo condicional, sin ser estadísticamente significativos. El cuadro 12 presenta los resultados de los tests LM que verifican la significación de esos residuos como variables omitidas en los dos modelos condicionales.

En todos los modelos marginales, se ha comprobado que sus residuos estimados no están correlacio- nados con los modelos condicionales respectivos. Según estos resultados, podemos decir que los parámetros de los modelos de corto plazo son invariantes, pues no están afectados por cambios de estructura en los modelos marginales. Por lo tanto, todas las variables condicionantes pueden admitirse como superexógenas, pues sus cambios de estructura no afectan los parámetros estimados, con lo que se soslaya la crítica de Lucas. $^{10}$

\section{Conclusiones}

El objetivo del presente trabajo es poner de manifiesto las principales variables determinantes de la inversión privada en Brasil durante el período 1956-1996. Partimos de un conjunto de variables explicativas basadas en los estudios empíricos más recientes para los países en desarrollo. Mediante el empleo de tests de estacionalidad, cointegración y exogeneidad, tratamos de obtener un modelo bien especificado, consistente y capaz de proporcionar informaciones relevantes para la conducción de políticas orientadas a incentivar la inversión privada.

Aunque las ecuaciones estimadas para el largo plazo hayan exhibido deficiencias por haber considerado algunas variables como exógenas, los dos modelos de regresión para el corto plazo presentaron regresores exógenos débiles y parámetros estructuralmente estables. Por lo tanto, podemos decir que los cambios estructurales en los modelos del producto, tipo de cam- bio, crédito, variación de la inflación e inversión pública no son responsables de los cambios estructurales en los modelos de la inversión privada, y que estos modelos no están sujetos a la crítica de Lucas.

Dado que los resultados obtenidos admiten la utilización de las variables explicativas de cada modelo como instrumentos de política, se ponen de manifiesto al menos tres formas de inducir un aumento de las inversiones del sector privado: i) aumento del nivel de actividad económica; ii) aumento del crédito y financiamiento de largo plazo, y iii) aumento de las inversiones en bienes públicos. Podemos decir, además, que

\footnotetext{
${ }^{10}$ Recurrimos también al análisis de los gráficos recursivos de los modelos marginales y condicionales, donde también se comprobó que los quiebres estructurales en los modelos de corto plazo no coinciden con los quiebres de los modelos marginales. Estos gráficos se omitieron por razones de espacio.
} 
durante los años noventa el análisis de indicadores relacionados con las variables producto, crédito e inversión pública, sería suficiente para explicar la caída de los niveles de inversión privada en Brasil.

Aparte de la influencia positiva del producto y aquella negativa de las condiciones de incertidumbre, en el caso brasileño se ha comprobado: i) la importancia de los créditos a largo plazo provenientes de los bancos de desarrollo; ii) el predominio del efecto de inducción (crowding-in) de la inversión privada que tiene la inversión pública, y iii) los impactos negativos de las desvalorizaciones cambiarias sobre la inversión. Así, las medidas que impliquen desvalorizaciones considerables del tipo de cambio o recortes de la inversión pública son perjudiciales para la formación de capital del país.

Por ende, podemos concluir que las sugerencias de políticas neoliberales del Consenso de Washington, respecto a reducir el papel del Estado en la asignación y creación de recursos (doctrina imperante entre quienes formulan las políticas a partir del segundo lustro de los años ochenta), son cuestionables. El repunte del crecimiento económico exige la participación del Estado, que tiende a estimular la expansión de la inversión privada, incluso en esta era de globalización.
Asimismo, el país necesita disponer de equilibrios fundamentales más sólidos de política económica, que comprendan: i) una tasa adecuada de interés real; ii) una tasa de inflación semejante a la de sus socios comerciales; iii) un tipo de cambio competitivo y previsible, y iv) estrategias de largo plazo para los proyectos de inversión pública. Tales metas deben ser consistentes con el equilibrio tanto interno como externo, a fin de hacer viable una política de crecimiento autosustentado, basado esencialmente en la inversión productiva.

Los modelos que hemos presentado pueden emplearse para derivar conclusiones de política económica. Con todo, cabe destacar que ellos omiten algunas cuestiones cruciales, como la no linealidad. Se puede argumentar que el proceso real de acumulación y desacumulación del capital, así como los efectos de la inversión sobre el ingreso, entrañan consideraciones relativas a la existencia o no de capacidad ociosa y la ocurrencia de crisis periódicas. Por tanto, se precisan sensibilidad y cautela para interpretar conclusiones econométricas en términos del proceso histórico de acumulación y crecimiento.

(Traducido del portugués)
Agosin, M.R. (1994): Saving and Investment in Latin America, Discussion papers, $N^{\circ} 90$, Ginebra, Conferencia de las Naciones Unidas sobre Comercio y Desarrollo (UNCTAD).

Caballero, R. (1993): On the dynamics of aggregate investment, en L. Servén y A. Solimano (eds.), Striving for Growth after Adjustment, Washington, D.C., Banco Mundial.

Charemza, W. y D. Deadman (1997): New Directions in Econometric Practice, Londres, Edward Elgar Publishing Ltd.

Cruz, B. De Oliveira y J.R. Teixeira (1999): Impacto de la inversión pública sobre la inversión privada en Brasil: 1947-1990, Revista de la Cepal, $\mathrm{N}^{\circ}$ 67, LC/G.2055-P, Santiago de Chile, Comisión Económica para América Latina y el Caribe (CEPAL).

Dickey, D. y W.A. Fuller (1981): Likelihood ratio statistics for autoregressive time series with a unit root, Econometrica, vol. 49, $\mathrm{N}^{\circ} 4$, Evanston, Illinois, The Econometric Society.

Engle, R.F. y C. Granger (1987): Co-integration and error correction: Representation, estimation and testing, Econometrica, vol. 55, $\mathrm{N}^{\circ} 2$, Evanston, Illinois, The Econometric Society.

Engle, R.F. y D.F. Hendry (1993): Testing super exogeneity and invariance in regression models, Journal of Econometrics, vol. $56, \mathrm{~N}^{\circ} 1 / 2$, Amsterdam, Países Bajos, North-Holland Publishing Company.

Engle, R.F., D.F. Hendry y J.F. Richard (1983): Exogeneity, Econometrica, vol. 51, $\mathrm{N}^{\circ}$ 2, Evanston, Illinois, The Econometric Society.

Greene, J. y D. Villanueva (1995): La inversión privada en los países en desarrollo: un análisis empírico, en A. Villagómez (comp.),
El financiamiento del desarrollo en América Latina: la movilización del ahorro interno, vol. 1, México, D.F., Centro de Estudios Monetarios Latinoamericanos (CEMLA).

Hendry, D. y G. Mizon (1993): Evaluating dynamic econometric models by encompassing the VAR, en P. Phillips (ed.), Models, Methods and Applications of Econometrics, Oxford, Reino Unido, Basil Blackwell.

Jogerson, D.W. (1963): Capital theory and investment behavior, The American Economic Review, vol. 53, N² 2, Nashville, Tennessee, American Economic Association, mayo.

Johansen, S. (1988): Statistical analysis of cointegration vectors, Journal of Economic Dynamics and Control, Nueva York, Elsevier, junio-septiembre.

(1994): Testing weak exogeneity and the order of cointegration in U.K. money demand data, N. Ericson y J. Irons (eds.), Testing Exogeneity, Oxford, Reino Unido, Oxford University Press.

Lucas, R. (1976): Econometric policy evaluation: A critique, K. Brunner y A. Meltzer (eds.), The Philips Curve and Labor Markets, vol. 1, Carnegie-Rochester Conferences on Public Policy, Amsterdam, Países Bajos, North Holland.

Perron, P. (1989): The great crash, the oil price shock, and the unit root hypothesis, Econometrica, vol. 57, $\mathrm{N}^{\circ} 6$, Evanston, Illinois, The Econometric Society.

Pindyck, R. (1988): Irreversible investment, capacity choice, and the value of the firm, The American Economic Review, vol. 78, $\mathrm{N}^{\circ}$ 5, Nashville, Tennessee, American Economic Association. 
Rocha, C.H. y J.R. Teixeira (1996): Complementaridade versus substituição entre investimento público e privado na economia brasileira: 1965-90, Revista brasileira de economia, vol. 50, $\mathrm{N}^{\circ}$ 3, Rio de Janeiro, Fundación Getulio Vargas.

Ronci, M.V. (1987): Política econômica e investimento privado no Brasil (1952/82), Rio de Janeiro, Escola de Pos-Graduação em Economia (EPGE)/Fundación Getulio Vargas, tesis de doctorado.
Servén, L. y A. Solimano (1992): Private investment and macroeconomic adjustment: A survey, The World Bank Research Observer, vol. 7, $\mathrm{N}^{\circ}$ 1, Washington, D.C., Banco Mundial.

(1993): Economic adjustment and investment performance in developing countries: The experience of the 1980s, L. Servén y A. Solimano (eds.), Striving for Growth after Adjustment, Washington, D.C., Banco Mundial. 